\title{
Associative-Commutative Discrimination Nets
}

\author{
Leo Bachmair Ta Chen I. V. Ramakrishnan \\ Department of Computer Science, SUNY at Stony Brook \\ Stony Brook, NY 11794, U.S.A.
}

\begin{abstract}
Use of discrimination nets for many-to-one pattern matching has been shown to dramatically improve the performance of the KnuthBendix completion procedure used in rewriting. Many important applications of rewriting require associative-commutative $(A C)$ function symbols and it is therefore quite natural to expect performance gains by using similar techniques for $A C$-completion. In this paper we propose such a technique, called $A C$-discrimination net, that is a natural generalization of the standard discrimination net in the sense that if no $A C$-symbols are present in the pattern, it specializes to the standard discrimination net. Moreover we show how $A C$-discrimination nets can be augmented so as to further improve the performance of $A C$-matching on problems that are typically seen in practice.
\end{abstract}

\section{Introduction}

Term matching is a fundamental operation in equational and functional programming and in various theorem proving methods, such as the many variants of the completion procedure [3]. In these applications the problem usually occurs in the form of many-to-one matching, where one has to determine for a given set of terms $t_{1}, \ldots, t_{n}$, also called patterns, if one matches a specified term $s$, called the subject. For instance, in a completion procedure the patterns to be considered are the left-hand sides of current rewrite rules, which may dynamically change.

The most efficient many-to-one matching algorithms use trie-like data structures, called discrimination nets, and corresponding tree automata, that allow one to factor out common expressions in a given set of patterns. Since completion procedures spend most of their time on normalization of terms (which consists of repeated steps of matching followed by term replacement), sophisticated matching algorithms based on discrimination nets may result in dramatic speedups, as has been demonstrated by Christian [1].

An important application of completion is to associative-commutative rewrite systems, which require a suitably modified operation, called associative-commutative matching (or $A C$-matching). One-to-one $A C$-matching is an $N P$-complete problem, but can be solved in polynomial time if patterns are restricted to linear terms (without multiple occurrences of variables) [2]. The essential component of the one-to-one $A C$-matching algorithm described by Benanav, Kapur, and Narendran [2] is the application of maximum bipartite graph matching. In fact, Verma and Ramakrishnan [13] showed that the two problems, associativecommutative matching and maximum bipartite graph matching are mutually 
reducible, so that complexity bounds for one problem also apply to the other. The best currently known lower bound for $A C$-matching is $O\left(m n^{1.5}\right)$, where $m$ refers to the size of the pattern and $n$ to the size of the subject.

In many applications, however, many-to-one matching is needed. Since $A C$ matching takes up most of the computation time in associative-commutative completion procedures - and discrimination nets had resulted in considerable speedups of standard completion-there have been attempts to adapt discrimination nets to $A C$-matching. These attempts have not been entirely satisfactory. In this paper, we demonstrate that discrimination nets, in combination with bipartite graph matching, can indeed be applied to do $A C$-matching efficiently. We propose associative-commutative discrimination nets, which are hierarchically structured collections of standard discrimination nets, with bipartite graph matching being used to combine the results from one level of the hierarchy so as to make them available to the next higher level. This approach applies to all different kinds of discrimination nets that have been proposed in the literature (deterministic or nondeterministic, adaptive or non-adaptive), and an associative-commutative net specializes to a standard discrimination net if applied to terms with no associative-commutative operators. The algorithm solves the many-to-one matching problem, but is of the same asymptotic complexity as the best current algorithm for the simpler problem of one-to-one matching.

The paper is organized as follows. In the next section we introduce some terminology and give a general definition of discrimination nets. In Section 3 we briefly discuss the reduction of associative-commutative matching to a matching problem on flattened terms. In Section 4 we introduce associative-commutative discrimination nets, design a corresponding matching algorithm, and analyse its complexity. In practical applications of associative-commutative matching, the bipartite graph matching problems that need to be solved are of a special form, which we exploit in Section 5 to improve the algorithm. Nonlinear matching is briefly discussed in Section 6 . In the concluding section we summarize our results and compare them to other recent work.

\section{Discrimination Nets}

We shall consider terms built from a given finite set of function symbols $\mathcal{F}$ and a (countable) set of variables $\mathcal{V}$. We use the symbols $s$ and $t$ to denote terms, $f$ and $g$ to denote function symbols, and $x, y$, and $z$ to denote variables. The arity of a function symbol $f$ is denoted by $\alpha(f)$. If $t$ is a term $f\left(t_{1}, \ldots, t_{n}\right)$, then $t_{1}, \ldots, t_{n}$ are called the top-level arguments of $t$.

The expression $\left.t\right|_{p}$ denotes the subterm of $t$ at position $p$. Positions may, for instance, be represented as sequences of integers. So, if $p^{\prime}=p q^{\prime}$ (that is, $p$ is a prefix of $p^{\prime}$ ), then $\left.t\right|_{p^{\prime}}$ is a subterm of $\left.t\right|_{p}$. We use $A$ for the top-most position $\left(\left.t\right|_{\Lambda}=t\right)$. By $t(p)$ we denote the symbol at position $p$ in $t$. The symbol $t(\Lambda)$ is also called the top-most, or root, symbol of $t$. For example, 2 is a position in $t=f(a, g(a, b))$ and $\left.t\right|_{2}=g(a, b)$, while $t(2)=g$. 
The application of a substitution $\sigma$ to a term $t$ is written $t \sigma$. A term $t$ is said to match another term $s$ (and $s$ is called an instance of $t$ ) if there is a substitution $\sigma$ such that $s=t \sigma$.

In term rewriting, efficient matching algorithms have been designed that employ an indexing technique similar to tries based on so-called discrimination nets. In defining discrimination nets, we have to refer to "partially constructed" terms. By a skeleton we mean a set $S$ of pairs $\left(p, f_{p}\right)$ of positions and function symbols, where (i) $S$ contains a pair with first component $p$ whenever it contains one with first component $p . i$, and (ii) if $p . i$ is a first component of some pair in $S$, then $i \leq \alpha\left(f_{p}\right)$. Given a (non-empty) skeleton $S$, we define its fringe to be the set of all positions $p . i$, such that $S$ contains a pair $\left(p, f_{p}\right)$, where $1 \leq i \leq \alpha\left(f_{p}\right)$, but contains no pair with first component p.i. The fringe of the empty skeleton is defined to be $\{\Lambda\}$. Skeletons and corresponding fringes can conveniently be represented as terms built from function symbols and some special constant $\square$ not contained in $\mathcal{F}$ : the fringe consists of all positions at which $\square$ occurs, while the remaining positions, with their corresponding function symbols, determine the skeleton. For example, the term $f(\square, g(a, \square))$ represents the skeleton $\{(\Lambda, f),(2, g),(2.1, a)\}$ with corresponding fringe $\{1,2.2\}$.

A term $t$ is said to be compatible with a skeleton $S$ if for every pair $\left(p, f_{p}\right)$ in $S$ either $p$ is a position in $t$ and $t(p)=f_{p}$, or else some prefix of $p$ is a variable position in $t$. A set $M$ of terms is said to be compatible with $S$ if each term in $M$ is. If $M$ is compatible with $S$, we say that $p$ is a discrimination position (for $M$ and $S$ ) if it is a position in the fringe of $S$ and $t(p)$ is a function symbol (not a variable), for some term $t$ in $M$.

Let $\boldsymbol{\omega}$ be a new constant not contained in $\mathcal{F}$. A matching tree is a tree where the edges are labelled by symbols from $\mathcal{F} \cup\{\omega\}$ and with each node $u$ is associated a non-empty set of terms $M_{u}$ (the match set), a skeleton $S_{u}$ (the partial match), and, if $u$ is not a leaf, a position $p_{u}$ (the discrimination position), such that (i) the empty skeleton is associated with the root of the tree; (ii) $p_{u}$ is a discrimination position for $M_{u}$ and $S_{u}$, for each non-leaf node $u$; and (iii) for each edge $(u, v), S_{v}=S_{u} \cup\left\{\left(p_{u}, f\right)\right\}$, where $f$ is the label of $(u, v)$, and $M_{v}$ is the set of all terms in $M_{u}$ that are compatible with $S_{v}$. Note that, for each node $u$, the match set $M_{u}$ is compatible with the skeleton $S_{u}$.

A discrimination net is a maximal matching tree that contains no duplicate nodes. (A node $v^{\prime}$ is a duplicate of another node $v$, if there are two edges $(u, v)$ and $\left(u, v^{\prime}\right)$ that are labelled by the same symbol.) In other words, a discrimination net is a matching tree to which no edges can be added. Thus, in a discrimination net there is no discrimination position for $M_{u}$ and $S_{u}$, for any leaf $u$. We also say that $D$ is a discrimination net for the set of terms $M$ associated with its root. Observe that, if $D$ is a discrimination net for $M$, all discrimination positions $p_{u}$ must be positions in some term $t$ of $M$. Furthermore the discrimination positions along any branch in $D$ are distinct, so that any discrimination net for a finite set of terms $M$ has to be finite.

The construction of a discrimination net for a given (finite) set of terms $T$ is straightforward. An initial matching tree consists of a single node $u$ labelled 
by $M_{u}=M$ and $S_{u}=\emptyset$. If for some leaf $v$ in a given matching tree there exists a discrimination position $p_{v}$ for $M_{v}$ and $S_{v}$, then the tree can be expanded correspondingly. If there are no further discrimination positions, the process terminates with a discrimination net. Since for any given leaf, there may be different discrimination positions to choose from, different discrimination nets may be obtained from a given initial set of terms $M$. In this sense our definition characterizes adaptive discrimination nets. Discrimination nets have typically been constructed according to some fixed order in which positions are chosen, e.g., left-to-right preorder as in [4]. The construction of discrimination nets can be optimized via a suitable choice of discrimination positions, in order to improve the matching time and decrease the size of the discrimination net, see [11]. A discrimination net for the set of terms $\{f(a, a), f(a, x)\}$ is shown in Figure 1(a).

Let $T$ be a set of linear terms (that is, terms without multiple occurrences of the same variable) and $D$ be a discrimination net for $T$. We define a corresponding matching automaton $A$ as follows. The nodes of $D$ are the states of $A$, with the root being the initial state and the leaves being final states. On a given input term $s$, the automaton makes a transition $u \rightarrow v$, if $D$ contains an edge $(u, v)$ labelled by the symbol $f$ and either $s\left(p_{u}\right)=f$ or else $s\left(p_{u}\right)$ is a variable and $f=\omega$. The automaton is said to succeed, if it reaches a final state, and is said to fail, otherwise.

We emphasize that these matching automata are deterministic (at any given moment an automaton may make at most one transition) and inspect each input symbol at most once. It can be proved that if an automaton reaches a final state $v$, then $M_{v}$ contains exactly those terms of $T$ which match $s$. If the automaton fails, then the input term $s$ is matched by no term in $T$. There are obvious optimizations of matching automata, such as merging equivalent states (which may be expressed in the discrimination net by labelling edges by sets of symbols); for further details see [11]. For example, Figure 1(b) shows an optimized version of the above net.

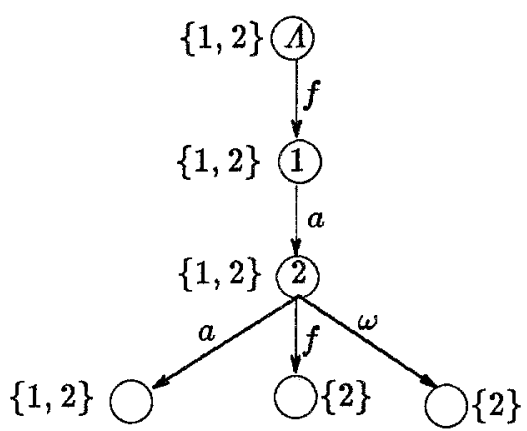

(a)

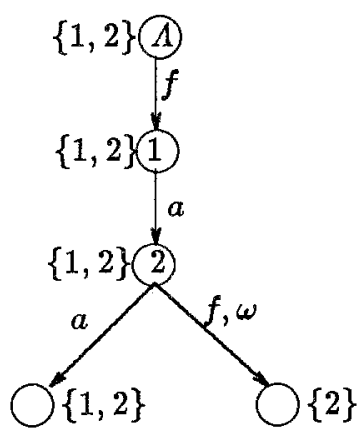

(b)

Fig. 1. (a) A discrimination net for $\{1: f(a, a), 2: f(a, x)\}$ and (b) its optimization. 
In dealing with nonlinear patterns one would first apply discrimination nets to linearized versions of the given terms, where different occurrences of the same variable are considered different, and in a second phase check whether the proposed instantiations for all occurrences of the same variable are consistent; see also Section 6.

It is possible to slightly change the definition of discrimination nets. We say that a term $t$ is strongly compatible with a skeleton $S$ if for every pair $\left(p, f_{p}\right)$ in $S, p$ is a position in $t$ and either $t(p)=f_{p}$ or else $t(p)$ is a variable and $f_{p}=\omega$. If $t$ is compatible with $S$, then some instance of $t$, but not necessarily $t$ itself, is strongly compatible with $S$. For example, both $f(x, a, b)$ and $f(b, a, a)$ are compatible with the skeleton $\{(\Lambda, f),(1, b)\}$, but only the second term is strongly compatible.

Let discrimination nets be defined as before, but with condition (iii) replaced by: (iii') for each edge $(u, v), S_{v}=S_{u} \cup\left\{\left(p_{u}, f\right)\right\}$, where $f$ is the label of $(u, v)$, and $M_{v}$ is the set of all terms in $M_{u}$ that are strongly compatible with $S_{v}$. Such discrimination nets can be used as nondeterministic automata, as follows. There is a transition $u \rightarrow v$ if $D$ contains an edge $(u, v)$ labelled either by the symbol $\omega$ or else by the symbol $f$, where $s\left(p_{u}\right)=f$. Thus, there may be different transitions from a state on the same input. Whenever a final state is reached, the corresponding match set contains only patterns that match the input term, but may not contain all such patterns. For further details see Christian [1]. Figure 2 illustrates the differences between the deterministic and nondeterministic versions of a discrimination net.

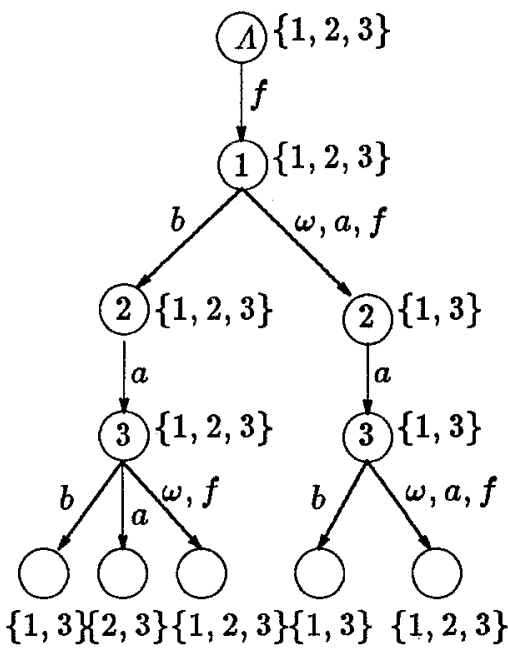

(a)

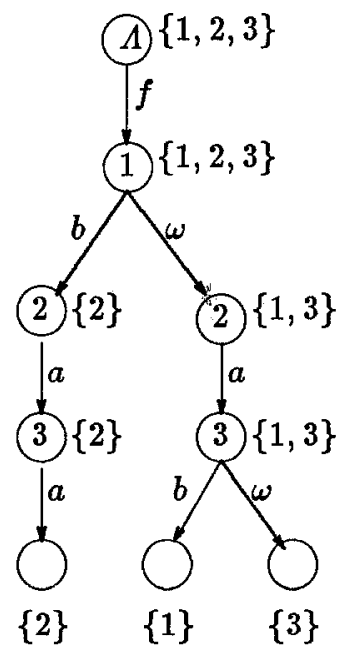

(b)

Fig. 2. Deterministic (a) and nondeterministic (b) discrimination nets for $\{1: f(x, a, b), 2: f(b, a, a), 3: f(x, a, y)\}$ 
Nondeterministic automata may require that all possible computation sequences be enumerated, so that the same symbol of the input term $s$ may have to be inspected repeatedly, as often as once for every pattern. Therefore they may be less efficient than deterministic automata in practice. On the other hand, they are smaller in size and can often be more easily updated, as patterns can readily be added to or deleted from $T$. Thus, in applications where the set of terms to match with keeps changing (such as in completion procedures), nondeterministic automata may be preferable, while for fixed sets $T$ deterministic automata are more efficient.

The associative-commutative matching algorithm proposed in this paper is based on the use of discrimination nets, but applies to any version thereof, whether deterministic or nondeterministic, adaptive or non-adaptive. For reasons of simplicity, our exposition is given in terms of deterministic automata.

\section{Associative-Commutative Matching}

Let $A C$ be a set of associativity and commutativity axioms

$$
\begin{aligned}
f(x, f(y, z)) & =f(f(x, y), z) \\
f(x, y) & =f(y, x)
\end{aligned}
$$

for some function symbols $f$. We write $f \in A C$ to indicate that $f$ is such an associative-commutative symbol, and denote by $={ }_{A C}$ the equational theory induced by the set of equations $A C$. Thus, $s={ }_{A C} t$ if and only if $s$ and $t$ are equivalent under associativity and commutativity.

A term $t$ is said to $A C$-match another term $s$ if there exists a substitution $\sigma$, such that $t \sigma={ }_{A C} s$. We shall first consider the problem of deciding, for given linear terms $t_{1}, \ldots, t_{n}$, which ones $A C$-match a specified term $s$.

In dealing with associativity and commutativity it is of advantage to "flatten" terms and allow varying arity for $A C$-symbols. More precisely, if $f$ is an $A C$ symbol, then $f(X)$ is a syntactically valid term, if the sequence of terms $X$ consists of at least two terms. (We denote the length of a sequence $X$ by $|X|$.)

By $L$ we denote the set of all rewrite rules (also called flattening rules) of the form

$$
f(X, f(Y), Z) \rightarrow f(X, Y, Z), \quad f \in A C,|X|+|Z| \geq 1,|Y| \geq 2 .
$$

Terms that can not be rewritten by $L$ are said to be flat. The normal form of a term $t$ under the flattening rules is denoted by $\bar{t}$. Let us denote by $\sim$ the smallest symmetric rewrite relation (also called the permutation congruence) for which

$$
f(X, u, Y, v, Z) \sim f(X, v, Y, u, Z), \quad \text { if } f \in A C .
$$

It is well-known that the flattened versions of terms equivalent under $A C$ are unique up to the permutation congruence. Consequently, a term $t A C$-matches a term $s$ if and only if there exists a substitution $\sigma$, such that $\overline{t \sigma} \sim \bar{s}$. If we assume that $x \sigma$ is a flat term, for all variables $x$, then $\bar{t} \sigma$ is almost a flat term as 
well. The only subterms to which a flattening rule can be applied are of the form $f\left(Y, x \sigma, Y^{\prime}\right)$, where $f \in A C$ and $x \sigma=f(Z)$, for some $A C$-symbol $f$, in which case $f\left(Y, f(Z), Y^{\prime}\right)$ will be flattened to $f\left(Y, Z, Y^{\prime}\right)$. The same effect is achieved by substituting for $x$, not the term $f(Z)$, but instead the sequence of terms $Z$. Denoting by $\bar{\sigma}$ this substitution, with sequences of terms substituted for variable arguments of $A C$-symbols, we find that $\bar{t} \sigma \sim \bar{s}$ is equivalent to $\bar{t} \bar{\sigma} \sim \bar{s}$.

In sum, the $A C$-matching problem can essentially be reduced to ordinary matching up to permutation of arguments of $A C$-symbols, provided terms are flattened first. Henceforth, we shall consider only flattened linear terms and speak of $A C$-matching to refer matching up to permutation. In the next section we apply discrimination nets to this problem.

\section{Associative-Commutative Discrimination Nets}

The $A C$-nesting depth at a position in a term is recursively defined as follows. The $A C$-nesting depth at $\Lambda$ is 0 . If $n$ is the $A C$-nesting depth at position $p$ in $t$, then the $A C$-nesting depth at a position $p . i$ in $t$ is $n+1$, if $t(p)$ is an $A C$-symbol, and $n$, otherwise. The $A C$-nesting depth of a term is the maximum $A C$-nesting depth of any of its positions. By the top-layer $\hat{t}$ of a term $t$ we mean the expression obtained from $t$ by removing all subterms at positions with nonzero $A C$-nesting depth. For example, if $f \in A C$ and $g \notin A C$, then the top-layer of $g(a, f(b, c))$ is $g(a, f)$. Observe that the top-layer of a term is a syntactically valid term if $A C$-symbols are regarded as constants. If $t$ contains no $A C$-symbols, then $\hat{t}=t$. Furthermore, if $s \sim t$, then $\hat{s}=\hat{t}$.

Now suppose $s$ and $t$ are flat terms, such that $t \sigma \sim s$, where $x \sigma$ may be a sequence of terms, if $x$ occurs as argument of an $A C$-symbol in $t$. Then (i) $\hat{t}$ matches $\hat{s}$ and (ii) for all positions $p$ of $A C$-nesting depth 0 in $t$, if $t(p)$ is an $A C$-symbol, then $\left.\left(\left.t\right|_{p}\right) \sigma \sim s\right|_{p}$. Conversely, if (i) and (ii) are satisfied for some substitution $\sigma$, then $t \sigma \sim s$. In other words, $A C$-matching can be characterized in terms of conditions (i) and (ii).

Condition (i) represents a standard matching problem. Let us consider condition (ii). Suppose $\left.t\right|_{p}=f\left(t_{1}, \ldots, t_{m}\right)$ and $\left.s\right|_{p}=f\left(s_{1}, \ldots, s_{n}\right)$, where $f \in A C$. Let us also assume, without loss of generality, that for some $k, 0 \leq k \leq m$, no term $t_{1}, \ldots, t_{k}$ is a variable, while all terms $t_{k+1}, \ldots, t_{m}$ are variables. Define a bipartite graph $G=\left(V_{1} \cup V_{2}, E\right)$, with $V_{1}=\left\{s_{1}, \ldots, s_{n}\right\}, V_{2}=\left\{t_{1}, \ldots, t_{k}\right\}$, and $E$ consisting of all pairs $\left(s_{i}, t_{j}\right)$, such that $s_{i} \sigma \sim t_{j}$, for some substitution $\sigma$. It can easily be seen that if (a) either $n=m$ or $n>m>k$, and (b) there is a matching of size $k$ in the bipartite graph $G$, then $f\left(t_{1}, \ldots, t_{m}\right) \sigma \sim f\left(s_{1}, \ldots, s_{n}\right)$, for some substitution $\sigma$ and hence condition (ii) is satisfied. (Recall that we consider only linear terms.)

In sum, a flat term $t A C$-matches another flat term $s$ if and only if (i) the top-layer of $t$ matches the top-layer of $s$ and (ii) maximal $A C$-subterms of $s$ are $A C$-matched by corresponding subterms of $t$. The second condition can be checked by $A C$-matching proper subterms of $t$ and $s$ and using bipartite graph matching. The above observations motivate the following definitions. 
If $T$ is a set of terms, we denote by $\hat{T}$ the set of all top-layers of terms in $T$. In a discrimination net for such a set $\hat{T}$, nodes $v$, for which there is an edge $(u, v)$ labelled by an $A C$-symbol, are called $A C$-nodes. Furthermore, we denote by $L_{v}$ the set of all terms $\left.t\right|_{p_{u}}$, for which $t \in T, \hat{t} \in M_{v}$, and $p_{u}$ is a non-variable position in $t$; and by $R_{v}$ the set of all non-variable terms that occur as top-level arguments of terms in $L_{v}$.

An $A C$-discrimination net is a hierarchically structured collection of standard discrimination nets. Formally, an $A C$-discrimination net for a set of flat terms $T$ consists of (i) a standard discrimination net $D$ (the top-level net) for the set $\hat{T}$ of top-layers of $T$, and (ii) associated with each $A C$-node $v$ in $D$, an $A C$-discrimination net (an $A C$-subnet) for the set $R_{v}$. By the depth of an $A C$ discrimination net for $T$ we mean the maximal $A C$-nesting depth of any term in $T$.

An $A C$-discrimination net for a set of terms $T$ defines an $A C$-matching automaton, which is deterministic and inspects each input symbol at most once, but differs from a standard matching automaton in that it dynamically computes current match sets.

The algorithm AC-match, which accepts as input a flat term $s$ and an associative-commutative discrimination net $D$ for a set of flat terms $T$, is defined as follows:

1. Let $u$ be the root of $D, D^{\prime}$ its top-level standard discrimination net, and $M_{u}^{\prime}$ be $T$.

2. If $u$ is leaf, then return $M_{u} \cap M_{u}^{\prime}$.

3. Otherwise, let $(u, v)$ be the edge in $D^{\prime}$ corresponding to the symbol $f=$ $s\left(p_{u}\right)$. (If $f$ is a variable, the corresponding edge is the one labelled by $\omega$.)

4. If $v$ is an $A C$-node, then $\left.s\right|_{p_{u}}$ is a term $f\left(s_{1}, \ldots, s_{n}\right)$. Recursively apply the algorithm AC-match to each term $s_{i}$ and the $A C$-subnet $D_{v}$ associated with $v$, to determine which terms in $R_{v} A C$-match $s_{i}$.

Define, for each term $t=f\left(t_{1}, \ldots, t_{k}, x_{k+1}, \ldots, x_{m}\right)$ in $L_{v}$ with non-variable arguments $t_{1}, \ldots, t_{k}$, a bipartite graph $G_{t}$ with vertices $s_{1}, \ldots, s_{n}$ and $t_{1}, \ldots$, $t_{k}$ and with all pairs $\left(s_{i}, t_{j}\right)$, for which $s_{i} A C$-matches $t_{j}$, as edges. If (a) either $n=m$ or else $n>m>k$, and (b) there is a matching of size $k$ in the graph $G_{t}$, then $t A C$-matches $f\left(s_{1}, \ldots, s_{n}\right)$.

Let $L_{v}^{\prime}$ be the set of all terms in $L_{v}$ that $A C$-match $f\left(s_{1}, \ldots, s_{n}\right)$. Let $M_{v}^{\prime}$ be the set of all terms in $M_{u}^{\prime}$, such that either $\left.t\right|_{p^{\prime}}$ is a variable, for some prefix $p^{\prime}$ of $p_{u}$, or else $\left.t\right|_{p_{u}}$ is in $L_{v}^{\prime}$.

5. Let $u$ be $v$ and $M_{u}^{\prime}$ be $M_{v}^{\prime}$ and go to step 2 .

This algorithm is correct:

Theorem 1. Let $D$ be an associative-commutative discrimination net for a set of flat terms $T$, and let $s$ be a flat term. Then the algorithm $\mathrm{AC}-$ match returns, for input $D$ and $s$, the set of all terms in $T$ that $A C$-match $s$.

Proof. The proof is by induction on the depth of $D$. Let $T^{\prime}$ be the set returned by AC-match for input $D$ and $s$ and let $t$ be a term in $T^{\prime}$. We first prove that (i) 
$\hat{t}$ matches $\hat{s}$ and (ii) for all positions $p$ of $A C$-nesting depth 0 in $t$, if $t(p)$ is an $A C$-symbol, then $\left.t\right|_{p} A C$-matches $\left.s\right|_{p}$.

Let $u$ be the root of the top-layer discrimination net $D^{\prime}$ of $D$ and let $v$ be the final state that is reached by the matching automaton $A^{\prime}$ defined by $D^{\prime}$ on input $\hat{s}$. The same node $v$ is the last one reached by AC-match. Since $t$ is in $M_{v}$, we may infer that $\hat{t}$ matches $\hat{s}$.

Let $p$ be a position of $A C$-nesting depth 0 in $t$, and suppose $\left.t\right|_{p}=f\left(t_{1}, \ldots, t_{m}\right)$ and $\left.s\right|_{p}=f\left(s_{1}, \ldots, s_{n}\right)$, for some $A C$-symbol $f$. Let $\left(u^{\prime}, v^{\prime}\right)$ be the edge considered in step 3 of AC-match at the time when the symbol at position $p$ in $s$ is inspected. Since any $A C$-subnet of $D$ is of smaller depth, we may use the induction hypothesis to infer that the set $M_{v}^{\prime}$ computed in the succeeding step 4 contains only terms $t^{\prime}$ such that $\left.t^{\prime}\right|_{p} A C$-matches $\left.s\right|_{p}$. Thus, conditions (i) and (ii) are indeed satisfied for $t$.

On the other hand, it can easily be seen that that if a term $t$ is contained in $T$ but not in $T^{\prime}$, then $t$ does not $A C$-match $s$. This completes the proof.

Theorem 2. Let $D$ be an associative-commutative discrimination net for a set of flat terms $T$, and let $s$ be a flat term. The algorithm AC-match determines the set of all terms in $T$ that $A C$-match $s$ in time $O(n)+O\left(m n^{1.5}\right)$, where $n$ is the size of the subject term $s$ and $m$ is the sum of the sizes of all pattern terms in $T$.

Proof. First note that each symbol in the input term $s$ is inspected at most once. If the symbol inspected is not $A C$, then its processing takes only a constant amount of time (steps 3 and 5 ).

In the case of an $A C$-symbol, which is the root of a subterm $f\left(s_{1}, \ldots, s_{n_{i}}\right)$ of $s$, we need to apply a bipartite graph matching algorithm to graphs $G_{t}$, for certain sets of patterns $t$, all of which are subterms of terms in the initial set $T$. Let $n_{i}+m_{t}$ be the number of vertices in $G_{t}$. Then the number of edges is at most $n_{i} m_{t}$, and a maximum bipartite matching on $G_{t}$ can be computed in time $O\left(m_{t} n_{i} \sqrt{n_{i}}\right)$. The total time for all bipartite matchings on graphs with $V_{1}=\left\{s_{1}, \ldots, s_{n_{i}}\right\}$ is thus no more than $O\left(m n_{i}^{1.5}\right)$. Since $\sum_{i} n_{i} \leq n$, the total time for all bipartite graph matchings done during the course of the algorithm is no more than $O\left(m n^{1.5}\right)$.

Furthermore, the computation of current match sets $M_{v}^{\prime}$ and returned match sets $M_{u} \cap M_{u}^{\prime}$ can be done in time proportional to the size of the sets involved, which is no more than $m$. Therefore the total time for all of these operations cannot exceed $O(n m)$. We have thus established the desired bound of $O(n)+$ $O\left(m n^{1.5}\right)$ on the running time of AC-match.

Finally, a few remarks about the space complexity of the discrimination net. The time to build the discrimination net is directly related to its space complexity, and the size of the discrimination net can become quite large. In fact, the worst-case lower bound on space of a discrimination net is exponential in the number of terms [11]. However, at the expense of increasing the matching time it is possible to reduce the space complexity. One approach is to build a nondeterministic net based on strong compatibility. Another approach that can yield 
much bigger space reductions is to build a different kind of discrimination net based on root-to-leaf path sequences which contain both positions and function symbols. Details about such nets appear in $[10,12,8]$.

\section{Secondary Automata}

In the above $A C$-matching algorithm we have applied indexing techniques to the non- $A C$ portions of given pattern terms, and have used bipartite graph matching to deal with $A C$-subterms. The running time of the algorithm is dominated by the cost of bipartite graph matching. The bipartite graphs that have to be considered are of the form $G=\left(V_{1} \cup V_{2}, E\right)$, where $V_{1}=\left\{s_{1}, \ldots, s_{l}\right\}$ is a set of input subterms, $V_{2}=\left\{t_{1}, \ldots, t_{k}\right\}$ is a set of pattern subterms, and $E$ contains ar edge $\left(s_{i}, t_{j}\right)$ if and only if $t_{j} A C$-matches $s_{i}$.

More precisely, the terms $t_{1}, \ldots, t_{k}$ are the non-variable top-level arguments of some occurrence of an $A C$-operator in a pattern term. It appears that in practice the number $k$ is usually quite small. For instance, in all the associativecommutative rewrite systems listed by Hullot [5], there are no $A C$-subterms with more than two non-variable arguments. We have also analysed several benchmark problems for $A C$-completion, such as certain group examples similar to the ones used by Christian [1]. In one typical example, a total of 128 rewrite rules were generated during the completion of a canonical system of 35 rules. There were 183 occurrences of $A C$-symbols on the left-hand sides of these rules, of which 81 had only variables as arguments, while the remaining ones had only one nonvariable argument. In the examples we looked at, there was only one instance of an $A C$-subterm with more than two non-variable arguments. (The number of non-variable arguments in that case was four.)

These observations have led us to design special techniques, called secondary automata, to efficiently handle bipartite graph matching for cases where $V_{2}$ is small, say $k \leq 4$.

Suppose graphs are represented by adjacency matrices, with rows corresponding to nodes $s_{i}$ and columns corresponding to nodes $t_{j}$. The edge set $E$ is computed row by row, as the algorithm $\mathrm{AC}$-match is applied to each term $s_{i}$. Let us denote by $G_{i}$ the subgraph of $G$ consisting of the first $i$ rows.

For example, suppose we have one pattern $f(g(a, b), g(a, x), y))$, where only $f$ is an $A C$-symbol. We need to look at bipartite graphs $G=\left(V_{1} \cup V_{2}, E\right)$, with $V_{2}=\{g(a, b), g(a, x)\}$. The sets $V_{1}$ and $E$ depend on the input term. For an input term $f(g(a, d), g(a, c), g(a, b))$, we have $V_{1}=\{g(a, d), g(a, c), g(a, b)\}$. The two terms $g(a, d)$ and $g(a, c)$ are $A C$-matched only by the second term in $V_{2}$, while $g(a, b)$ is $A C$-matched by both pattern subterms, so that we obtain adjacency matrices

$$
G_{1}=\left(\begin{array}{ll}
0 & 1
\end{array}\right), G_{2}=\left(\begin{array}{ll}
0 & 1 \\
0 & 1
\end{array}\right), G=G_{3}=\left(\begin{array}{ll}
0 & 1 \\
0 & 1 \\
1 & 1
\end{array}\right) .
$$

The maximum graph matching is of size 2 . 
Let us classify bipartite graphs $G=\left(V_{1} \cup V_{2}, E\right)$ with $\left|V_{2}\right|=k$, according to the size of a maximum matching and the subset of vertices in $V_{2}$ matched in a maximum matching. More precisely, a matching characteristic is a collection $\delta$ of $d$-element subsets of $V_{2}$, where $d \leq k$. We say that $G$ has matching characteristic $\delta$ if, for every set $M \in \delta$, there exists a maximum matching of $G$ on which each vertex in $M$, but no vertex in $V_{2} \backslash M$, is incident. (Thus, the size of the maximum matching is $d$.) Note that there are only finitely many different sets $\delta$, for any fixed number $k$.

For example, graphs $G_{1}$ and $G_{2}$ above have matching characteristic $\{2\}$, while $G_{3}$ has matching characteristic $\{1,2\}$.

We define an automaton $S_{k}$, for each $k$, as follows. Each matching characteristic $\delta$ is represented by a unique state of $S_{k}$. The input symbols for $S_{k}$ are bitstrings $b$ of length $k$ (each such bitstring representing a possible row in adjacency matrix). There is a transition from $\delta$ to $\delta^{\prime}$ on input $b$ if there is some graph $G$ of matching characteristic $\delta$ and with adjacency matrix $A$, such that the graph $G^{\prime}$ defined by the adjacency matrix obtained from $A$ by adding row $b$, has matching characteristic $\delta^{\prime}$. There is exactly one state $\delta_{0}$ with $\left|\delta_{0}\right|=0$, which is taken as the initial state of $S_{k}$; and exactly one state $\delta_{k}$ with $\left|\delta_{k}\right|=k$, which is taken as the only final state of $S_{k}$. We call $S_{k}$ a secondary automaton.

The secondary automata $S_{2}$ is shown in Figure 3. The states are numbered, with matching characteristic as indicated. For instance, state 3 has matching characteristic $\{\{1\},\{2\}\}$, which is the property of any graph $G=\left(V_{1} \cup V_{2}, E\right)$, where $V_{2}$ has two elements, $E$ is non-empty, and all edges are incident on the same element (either the first or the second) of $V_{2}$.

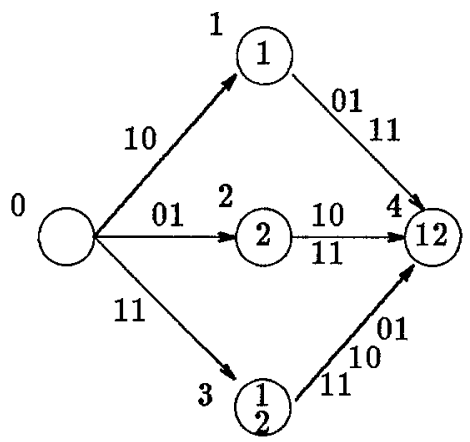

Fig. 3. Secondary automaton of depth 2.

Observe that all transitions lead from a state representing matchings of size $d$ to a state representing matchings of size $d+1$. In fact, we have:

Lemma 3. Let $G=\left(V_{1} \cup V_{2}, E\right)$ and $G^{\prime}=\left(V_{1}^{\prime} \cup V_{2}, E^{\prime}\right)$ be bipartite graphs, such that $V_{1}^{\prime}=V_{1} \cup\{v\}$ and $E^{\prime}=E \cup E_{v}$, where $E_{v}$ is a set of edges $\left(v, v^{\prime}\right)$, with $v^{\prime} \in V_{2}$. Also, let d be the size of a maximum matching in $G$. Then $G^{\prime}$ has either 
a maximum matching of size $d+1$, or else has the same matching characteristics as $G$.

Proof. Let the matching characteristics of $G$ and $G^{\prime}$ be $\delta$ and $\delta^{\prime}$ respectively. Suppose $G^{\prime}$ has a maximum matching of size $d, \delta \neq \delta^{\prime}$ and $M^{\prime} \in \delta^{\prime} \backslash \delta$. For any $M \in \delta$, there must be a vertex $w \in M^{\prime}$ such that $w \notin M$. Clearly, $v$ is matched with a vertex $u \in M$ because otherwise $M^{\prime}$ would be either of size $d+1$ or included in $\delta$. Let $v^{\prime}$ be the vertex matched with $u$ in a matching characterized by $M$. If $v^{\prime}$ is not matched with any vertex in $M^{\prime}$, then $M^{\prime} \in \delta$, which is a contradiction. Suppose $u^{\prime}$ is $v^{\prime \prime}$ 's match in $M^{\prime}$. If $u^{\prime} \in M$, one can repeat the above argument until a vertex $w \in V_{2}$ but $w \notin M$ is found; otherwise, we take $w=u^{\prime}$. In both cases, we have shown for $G^{\prime}$ the existence of maximum matchings of size $d+1$ incident upon the subset $M \cup\{w\}$ of $V_{2}$. A contradiction.

As an immediate corollary we obtain:

Corollary 4. Let $G=\left(V_{1} \cup V_{2}, E\right)$ be a bipartite graph with $\left|V_{1}\right|=l$ and $\left|V_{2}\right|=$ $k$. Let $A$ be the corresponding adjacency matrix, and $A_{i}$ be the $i$-th row of $A$. Then the secondary automaton $S_{k}$, for input $A_{1} \ldots A_{l}$, will reach the state $\delta$ that represents the matching characteristics of $G$.

In the context of the algorithm AC-match the secondary automata can be used in step 4, to determine whether there exists a bipartite matching of the required size. The number of states in a secondary automaton increases exponentially with $k$, so that these automata are only practical for small values of $k$. (The automaton $S_{1}$ has only two states, while $S_{2}$ has five, and $S_{3}$ sixteen.) Fortunately, the value of $k$ tends to be small enough in practical examples, as we have mentioned above.

Secondary automata can also be adapted to yield the actual matching substitutions. If, in traversing a secondary automaton, one can not move to a new state on a row of the adjacency matrix, the input subterm corresponding to the row is a candidate term for the substitution of some variable $x_{k+1}, \cdots, x_{m}$. By keeping track of such candidates, once the $A C$-matching is done, one can find consistent substitutions for all the variables in a pattern. For instance, in the pattern $f(g(a, x), g(b, y))$ and the subject $f(g(a, b), g(b, c))$, where both $f$ and $g$ are in $A C, y$ has two candidate substitutions $a$ and $c$ while $x$ has only $b$. From these candidates, one can determine the final substitution $x \mapsto b$ and $y \mapsto c$ once the algorithm has finished.

Theorem 5. All bipartite matching problems at all AC-nodes visited during traversal of an AC-discrimination net for a set of flat terms, with total size $m$, on behalf of an input term of size $n$ can be done in $O(m n)$ time.

Proof. Since the transition function at each state of a secondary automaton of depth $k$ can be implemented as a $k$-dimensional table, each transition takes $O(k)$. Thus, using the notation as in proof of Theorem 2, a maximum bipartite matching on $G_{t}$ can be computed in time $O\left(m_{t} n_{i}\right)$. Therefore, the time to compute maximum bipartite matchings on graphs with $V_{1}=\left\{s_{1}, \cdots, s_{n_{i}}\right\}$ is no more than $O\left(m n_{i}\right)$ and the total time for all bipartite matchings done during the course of AC-match is no more than $O(m n)$. 


\section{Nonlinear Matching}

The above (polynomial-time) algorithm applies to linear pattern terms. In its simplest form, the algorithm just determines which patterns $A C$-match a given input term. In situations where most match attempts are likely to fail (as is typical of theorem proving applications), it may thus make sense to use this version of the algorithm as a "filter," to quickly sort out failed matching attempts. In case of success, a single substitution for a pattern can easily be extracted during the algorithm, which is sufficient for the purpose of rewriting with linear terms.

In the case of nonlinear terms, we apply the matching algorithm to linearized versions of patterns in a first phase, and, in a second phase, check whether the proposed substitutions for different occurrences of the same variable are consistent. This requires that all matching substitutions be computed, and the $A C$-matching algorithm has to be extended correspondingly. In particular, we have to compute all maximal bipartite graph matchings-instead of just determining whether a maximum-sized matching exists. (The nonlinear $A C$-matching problem is $N P$-complete, so that a considerable increase in computing time, as compared to linear $A C$-matching, is not surprising.)

\section{Concluding Remarks}

In this paper, we have proposed a new data structure, called $A C$-discrimination nets, and used it to speed up many-to-one $A C$-matching. In any set of patterns the nesting depth of $A C$-symbols defines a number of levels of non- $A C$-parts. An $A C$-discrimination net is a collection of standard discrimination nets, structured in a way that reflects this hierarchy. The $A C$-matching algorithm, recursively traversing the levels of the net, uses bipartite graph matching on each level to interpret and combine the $A C$-matching results from lower levels. If no $A C$ symbols are present in the patterns, the $A C$-discrimination net specializes to a standard discrimination net.

We have also proposed secondary automata as a novel data structure for further speeding up matching of $A C$-patterns typically encountered in practice. Secondary automata are only dependent on the number of non-variable arguments within $A C$-subterms of the pattern, so that all $A C$-subterms having the same number of non-variable arguments can be handled by a single secondary automaton. This feature makes it attractive for completion as there is no need to create them dynamically when patterns change.

Let us briefly discuss some of the other proposals for many-to-one $A C$ matching. Lugiez and Kounalis [6] proposed an algorithm to compile pattern matching of $\mathrm{AC}$ function definitions in functional languages. In their approach a complete pattern tree (that guides matching) is constructed by enumerating certain AC terms. Such an enumeration appears to be prohibitively expensive in terms of both time and space. Nicolaita [9] described a method. which is based on Christian's discrimination net [1]. The main drawback with his method is that it requires that the arguments of $A C$-subterms in the patterns as well as in 
the subject be ordered. It is not clear whether the ordering constraints can be efficiently maintained in completion, where both the patterns and the subjects keep changing dynamically. (The kind of ordering to be used is left unspecified and the complexity of the algorithm is left open.) Lugiez and Moysset describe a bottom-up procedure for $A C$-matching in which patterns are preprocessed into a large nondeterministic tree automaton [7]. The running time of the algorithms is not analysed, but the construction of a deterministic automaton (from the nondeterministic one) is bound to be expensive.

Finally we remark that our linear matching algorithm can be directly used to deal with function symbols that are only commutative, in which case no flattening rules are applied to such function symbols. For function symbols that are only associative we simply need to replace bipartite graph matching with ordered bipartite matching.

Acknowledgments. We wish to thank the referees for their comments. This research has been supported in part by NSF grant CCR-9102159.

\section{References}

1. J. Christian. Fast Knuth-Bendix completion : Summary. In RTA'89, pages 551555. Springer-Verlag LNCS 355, 1989.

2. D. Kapur D. Benanav and P. Narendran. Complexity of matching problems. Journal of Symbolic Computation, 3:203-216, 1987.

3. N. Dershowitz and J.-P. Jouannaud. Rewrite systems. In Handbook of Theoretical Computer Science, volume B, chapter 6, pages 243-309. Elsevier, 1990.

4. A. Graf. Left-to-right tree pattern matching. In $R T A$ '91, pages 323-334. SpringerVerlag LNCS 488, 1991.

5. J.-M. Hullot. A catalogue of canonical term rewriting systems. Technical Report CSL-113, SRI International, April 1980.

6. E. Kounalis and D. Lugiez. Compilation of pattern matching with associativecommutative functions. In TAPSOFT'91, pages 57-73. Springer-Verlag LNCS 493, 1991.

7. D. Lugiez and J.L. Moysset. Complement problems and tree automata in AC-like theories. To appear in STACS'g3.

8. W. McCune. Experiments with discrimination-tree indexing and path indexing for term retrieval. Technical Report MCS-P191-1190, Argonne National Laboratory, Argonne, Mlinois, USA, January 1991.

9. D. Nicolaita. An indexing scheme for AC-equational theories. Technical report, Research Institute for Infomatics, Bucharest, Romania, February 1992.

10. R. Ramesh, I.V. Ramakrishnan, and D.S. Warren. Automata-driven indexing of prolog clauses. In Seventh Annual ACM Symposium on Principles of Programming Languages, pages 281-290, San Francisco, 1990.

11. R.C. Sekar, R. Ramesh, and I.V. Ramakrishnan. Adaptive pattern matching. In ICALP'92, pages 247-260. Springer-Verlag LNCS 623, 1992.

12. M. E. Stickel. The path-indexing method for indexing terms. Technical Report 473, SRI International, Menlo Park, California, USA, October 1989.

13. R. M. Verma and I.V. Ramakrishnan. Tight complexity bounds for term matching problems. Information and Computation, 101(1):33-69, November 1992. 